\title{
Functional somatic syndromes: asking about exclusionary medical conditions results in decreased prevalence and overlap rates
}

\author{
Susanne Fischer ${ }^{*}$ and Urs M Nater
}

\begin{abstract}
Background: The diagnosis of functional somatic syndromes (FSS) requires 1) presence of somatic symptoms, and 2) absence of medical conditions potentially accounting for these symptoms. Due to the limited feasibility of medical examinations, epidemiological research on FSS has neglected to assess the second criterion. Our objective was therefore to evaluate the implications of considering information on exclusionary medical conditions in epidemiological research on FSS.

Methods: A survey among 3'054 students was conducted. We compared prevalence rates and overlap of 17 FSS obtained by: 1) a symptom-based strategy and 2) a symptom-and-exclusion-based strategy including information on exclusionary medical conditions.

Results: The symptom-and-exclusion-based strategy led to a marked decrease in prevalence rates compared to the symptom-based strategy. Furthermore, it resulted in fewer individuals who were affected by multiple FSS.

Conclusions: Adding self-reported information on exclusionary medical conditions leads to a significant decrease in the prevalence and overlap of FSS. More rigorous approaches to studying FSS should be adopted.
\end{abstract}

Keywords: Diagnostic criteria, Epidemiology, Functional somatic syndromes, Overlap, Population-based, Prevalence

\section{Background}

The term 'functional somatic syndrome' (FSS) refers to a certain constellation of somatic symptoms that cannot be adequately explained in the context of a known medical condition. Case definitions of the numerous existing FSS therefore each require 1) the presence of at least one characteristic symptom (positive criterion), and 2) the absence of any medical condition that can account for these symptoms (negative criterion). There is a long list of FSS, but among the most prevalent are chronic fatigue syndrome, fibromyalgia syndrome, and irritable bowel syndrome. The diagnostic criteria for FSS are commonly formulated by expert committees; examples are the 1994 Centers for Disease Control and Prevention criteria for chronic fatigue syndrome [1], the Rome III criteria for irritable bowel syndrome (and other functional gastrointestinal disorders)

\footnotetext{
* Correspondence: susanne.fischer@uni-marburg.de

Clinical Biopsychology, Department of Psychology, University of Marburg, Gutenbergstrasse 18, 35032 Marburg, Germany
}

[2], and the 1990 and 2010 American College of Rheumatology criteria for fibromyalgia $[3,4]$.

These diagnostic criteria are used in clinical practice and research settings, where patients are asked about symptoms (positive criterion), medical records are reviewed, and physical examinations and laboratory tests are performed in order to identify medical conditions considered exclusionary for FSS (negative criterion). This two-step approach, which covers the assessment of both criteria inherent in the definition of FSS, is considered the gold standard for diagnosing an FSS. However, epidemiological research is challenged by the limited feasibility of reviewing medical records and/or conducting comprehensive medical examinations, and thus often exclusively relies on self-reported information. Several ways of diagnosing FSS have been adopted to deal with this problem: a) asking patients whether they suffer from a (specific) FSS (self-reported diagnosis), b) asking patients whether they have ever received an FSS diagnosis by a physician (physician diagnosis), or c) providing patients with a list of 
characteristic symptoms in accordance with the diagnostic criteria, but without an assessment of exclusionary factors (symptom-based diagnosis). Naturally, the approaches leading to these outcomes differ in their ability to cover both the positive and negative criterion of FSS.

It is conceivable from this comparison that the choice of diagnostic strategy may contribute to diverging study findings. In fact, reviews on the epidemiology of each FSS show a broad range of prevalence rates across studies (e.g., [5-7]). Another epidemiological estimate rather specific to research on FSS is the amount of comorbidity among FSS, i.e., the so-called 'overlap'. With regard to prevalence rates, overlap between FSS has been found to vary substantially $[8,9]$. Importantly, studies showing high levels of overlap have led some researchers to propose the existence of only one FSS [10]. These so-called 'lumpers' are opposed by other authors, who insist that there are several specific syndromes, and these authors are usually referred to as 'splitters' [11]. Thus, the overlap rates can be considered a key parameter in the so-called 'one vs. many debate'. However, direct evidence on the repercussions of using different diagnostic strategies as a possible reason for the observed discrepancies in prevalence rates and overlap is extremely scarce.

To the best of our knowledge, so far, only one study has directly examined the consequences of using different diagnostic strategies for FSS. In a recent study conducted among female FSS patients and matched controls, Warren and Clauw [12] reported a lack of sensitivity and specificity of physician diagnoses (the above-mentioned option b) when compared to symptom-based diagnoses (option c). While we fully agree with the authors' conclusion that 'queries of symptoms, not diagnoses, are necessary' (p. 894 in the same article), we believe that merely asking about characteristic symptoms (positive criterion) may result in an overestimation of FSS prevalence (and possibly overlap) rates. In cases in which a thorough medical examination is not feasible (such as in the above-mentioned study designs), we believe it preferable to also obtain self-reported information on medical illnesses considered exclusionary for FSS (negative criterion). In essence, we would argue in favor of a combination of options b) and c) in determining FSS diagnoses in epidemiological studies (symptom-andexclusion-based strategy).

However, the potential impact of this strategy needs to be examined. We aimed to extend the findings reported by Warren and Clauw [12] by comparing two different diagnostic strategies in 17 different FSS in a large, nonclinical sample of young adults. The two strategies were as follows: 1) identifying cases of FSS by means of presenting a list of symptoms that are based on the diagnostic criteria (symptom-based strategy), and 2) additionally asking about medical exclusionary criteria (symptom-and-exclusion-based strategy). We expected to find 1) a significant decrease in prevalence rates of FSS, and 2) a marked decrease in the extent of overlap between FSS when using the symptom-and-exclusionbased strategy. We tested these hypotheses as part of a larger study on the prevalence, overlap, and predictors of FSS [13].

\section{Methods}

\section{Participants}

The recruitment procedure for participants in this study has been described previously elsewhere [13]. In brief, German-speaking students from 23 Swiss colleges and universities were contacted via e-mail through cooperating school administrators, and asked to participate in a web survey on physical and mental well-being. All procedures were in accordance with the ethical standards laid down in the 1964 Declaration of Helsinki, and the web survey study design was approved by the ethics committee of the Canton of Zurich. Written informed consent was obtained from all participants.

\section{Measurement}

We administered a previously developed questionnaire (Questionnaire on Functional Somatic Syndromes; FFSS; [14]). The German version of the FFSS is freely available as a Web supplement to the original article (http://content.karger.com/ProdukteDB/miscArchiv/000/333/298/ 000333298_sm_supplemental_material.pdf). The FFSS consists of three different parts which are connected via several algorithms. In the first part, a screening section encompassing 52 items on various somatic symptoms was presented. These items represent cardinal symptoms of 17 FSS: Tension-type headache and persistent idiopathic facial pain [15], whiplash-associated disorders (pain of at least 6 months' duration that is related to an accident), temporomandibular disorders [16], globus and functional chest pain of presumed esophageal origin [17], functional dyspepsia [18], irritable bowel syndrome [2], chronic low back pain (lower back pain of at least 6 months' duration causing impairment), fibromyalgia syndrome [3], chronic fatigue syndrome [1], multiple chemical sensitivity [19], chronic pelvic pain in men [20] and in women (lower abdominal pain of at least 6 months' duration), premenstrual syndrome [21] and premenstrual dysphoric disorder [22], and hyperventilation syndrome [23]. The instruction was to rate all current symptoms ('I suffer from the following complaints:') according to frequency of occurrence ('never/rarely', 'frequently', 'almost always/always'). In addition, the screening part contains dichotomous questions on functional impairment due to symptoms in different areas and a categorical item on the duration of symptoms.

In the second part, if participants reported cardinal symptoms that were at least 'frequently' present and 
characteristic of one of 17 FSS (e.g., abdominal pain in the case of irritable bowel syndrome), additional questions based on diagnostic criteria (e.g., Rome III) were presented. Our questions were based on the most commonly used diagnostic criteria (all publications containing these criteria can be found in the previous section for each FSS). These questions allowed for a detailed understanding of both cardinal and associated symptoms, symptom course and fluctuation, functional impairment, and symptom onset for each FSS. Participants were labelled as having a 'symptom-based FSS' if they met the minimum of required positive criteria (e.g., recurrent abdominal pain or discomfort on at least 3 days per month in the last 3 months including changes in bowel movement, with symptom onset at least 6 months previously).

In the third part, those who fulfilled the positive criteria of a specific FSS were subsequently surveyed about health care visits. Importantly, visits related to the previously diagnosed FSS (but not health care visits in general) were of interest at this point (e.g., 'Have you ever visited a doctor about your abdominal pain/changes in bowel movement?'). Participants who responded with 'yes' were ultimately directed to a list of items addressing frequent differential diagnoses ("What diagnosis did your doctor give you regarding your abdominal pain/ changes in bowel movement?'). These lists were again based on the diagnostic criteria for each FSS as cited above. If they reported that no abnormalities had been detected by their doctor that might account for their symptoms (e.g., an inflammatory bowel disease), participants were labelled as having a symptom-andexclusion-based FSS'. The FFSS screening part has good psychometric properties regarding both internal consistency (Cronbach's alpha $=0.94$ ) and retest reliability $(r=0.80-0.94)$.

Prevalence rates and overlap estimations of symptomand-exclusion-based FSS have already been described in our previous report [13].

\section{Results}

\section{Participants' characteristics}

Our recruitment and data preparation process is visualized in Figure 1. A total number of $N=6,206$ participants visited the website and about $51 \%$ of them finished the survey. After the exclusion of implausible and incomplete datasets (regarding survey response duration, gender, and age), $\mathrm{N}=3$ '054 datasets remained for further analyses. Out of these 3'054 participants, 2'242 (73.4\%) were women and 812 (26.6\%) were men. The mean age was $24.6 \pm 5.6$ (SD) years. Parental household income was almost uniformly distributed across nine predefined categories ranging from less than 3'000 to more than 10'000 Swiss Francs per month (equal intervals across categories).

\section{Prevalence of FSS}

As illustrated in Figure 1, about one third of our sample endorsed an FSS when using the symptom-based strategy. Half of these participants had embarked upon health care visits because of their symptoms. More than half of the health care visitors were not offered a medical explanation for their symptoms and those were thus labelled symptom-and-exclusion-based FSS cases. To compare the impact of our two diagnostic strategies on epidemiological data, we calculated the prevalence rates of 17 FSS according to both strategies. We additionally included the health care visitor data for descriptive purposes. No male participant reported suffering from chronic pelvic pain and thus this FSS was excluded from all analyses. The results are illustrated in Figure 2. The prevalence rates of the premenstrual syndrome, premenstrual dysphoric disorder, and chronic pelvic pain all refer to the female population only. In accordance with our first hypothesis, we observed marked decreases in prevalence rates when using a symptom-and-exclusion-based approach to diagnosing FSS.

\section{Overlap between FSS}

To evaluate the potential impact of our two diagnostic strategies on the extent of overlap between FSS, we counted the number of FSS per person according to each strategy. The number of symptom-based FSS per person ranged from one to eight, with 631 (62.2\%) participants reporting only one, 239 (23.6\%) reporting two, $92(9.1 \%)$ reporting three, 35 (3.5\%) reporting four, 13 (1.3\%) reporting five, three $(0.2 \%)$ reporting six, and one person (0.1\%) reporting eight symptombased FSS occurring at the same time. The number of symptom-and-exclusion-based FSS ranged from one to four: 227 (78.5\%) participants fulfilled criteria for only one, 49 (17.0\%) reported two, 12 (4.2\%) reported three, and one person (0.3\%) reported four symptom-and-exclusion-based FSS simultaneously.

We then calculated the number of co-occurring FSS for each strategy separately. Since premenstrual syndrome represents a less severe form of premenstrual dysphoric disorder, the extent of overlap between these syndromes was not evaluated. We first looked at each FSS separately. For instance, within the irritable bowel syndrome group, most people had one additional FSS, but some had up to seven additional syndromes. We did this with every syndrome and computed an average index. Within the symptom-based FSS group, $9.2 \pm 3.6$ different co-occurring syndromes (out of 16) were present on average, whereas individuals with symptom-and-exclusion-based FSS fulfilled criteria for an average amount of $3.7 \pm 3.0$ co-occurring syndromes (out of 15 , excluding idiopathic facial pain). 


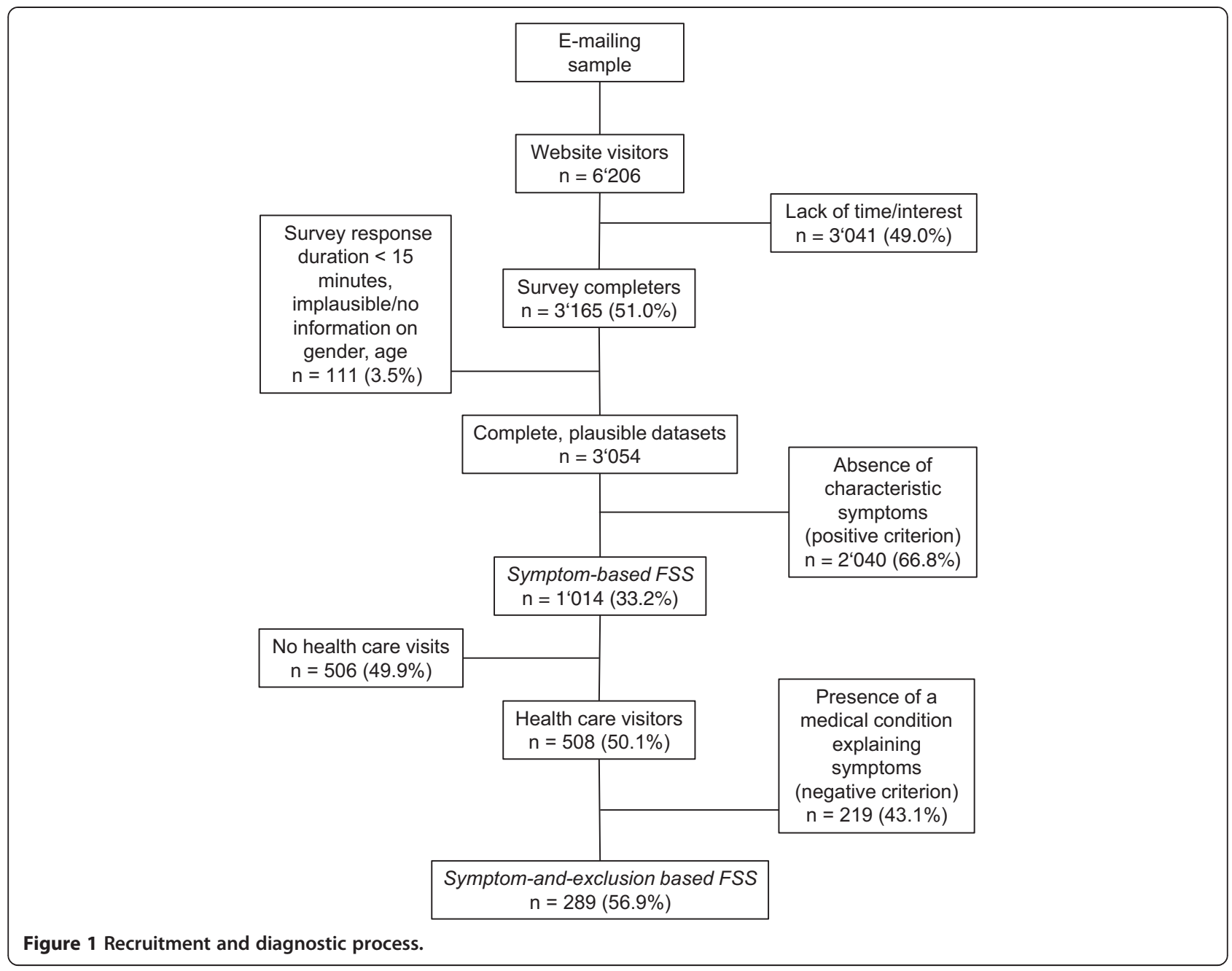

\section{Discussion}

\section{Summary of study results}

In this study, we aimed to evaluate the implications of considering self-reported information on exclusionary medical conditions in epidemiological research on FSS. We compared prevalence rates and overlap of 17 FSS diagnoses obtained by two different diagnostic strategies: a symptom-based strategy and a symptom-and-exclusionbased strategy. We report two findings that are in accordance with our initial hypotheses: First, the use of medical exclusionary criteria (symptom-and-exclusion-based strategy) led to a marked decrease in prevalence rates of FSS when compared to the symptom-based strategy. Second, the use of the symptom-and-exclusion-based strategy resulted in fewer numbers of individuals who were affected by multiple FSS at the same time. Moreover, it also resulted in fewer overlapping syndromes.

\section{Integration and interpretation of study results}

This is the first study to directly examine the impact of adding information on exclusionary medical conditions on the prevalence of FSS. In a recent report, Warren and Clauw [12] found symptom-based diagnoses to be superior to physician diagnoses of FSS in terms of sensitivity and specificity. While this is an important finding, with both diagnostic and clinical implications, the results of the present study indicate that a symptom-based strategy might, in turn, overestimate prevalence rates of FSS. This is most likely due to a participant's incorrect attribution of a somatic symptom (e.g., abdominal pain) to a specific FSS (e.g., irritable bowel syndrome), when, in fact, it is part of a medical illness (e.g., Crohn's disease).

Our finding of a marked decrease in prevalence rates of FSS when considering exclusionary medical conditions is mirrored by other population-based research adopting the gold standard procedure, in which patients are first asked about symptoms (positive criterion), followed by physical examinations and laboratory testing (negative criterion). None of these studies explicitly assessed the ramifications of using different diagnostic strategies; however, their detailed reporting of patient screening procedures (e.g., using flow charts) allows the reader to compare the 


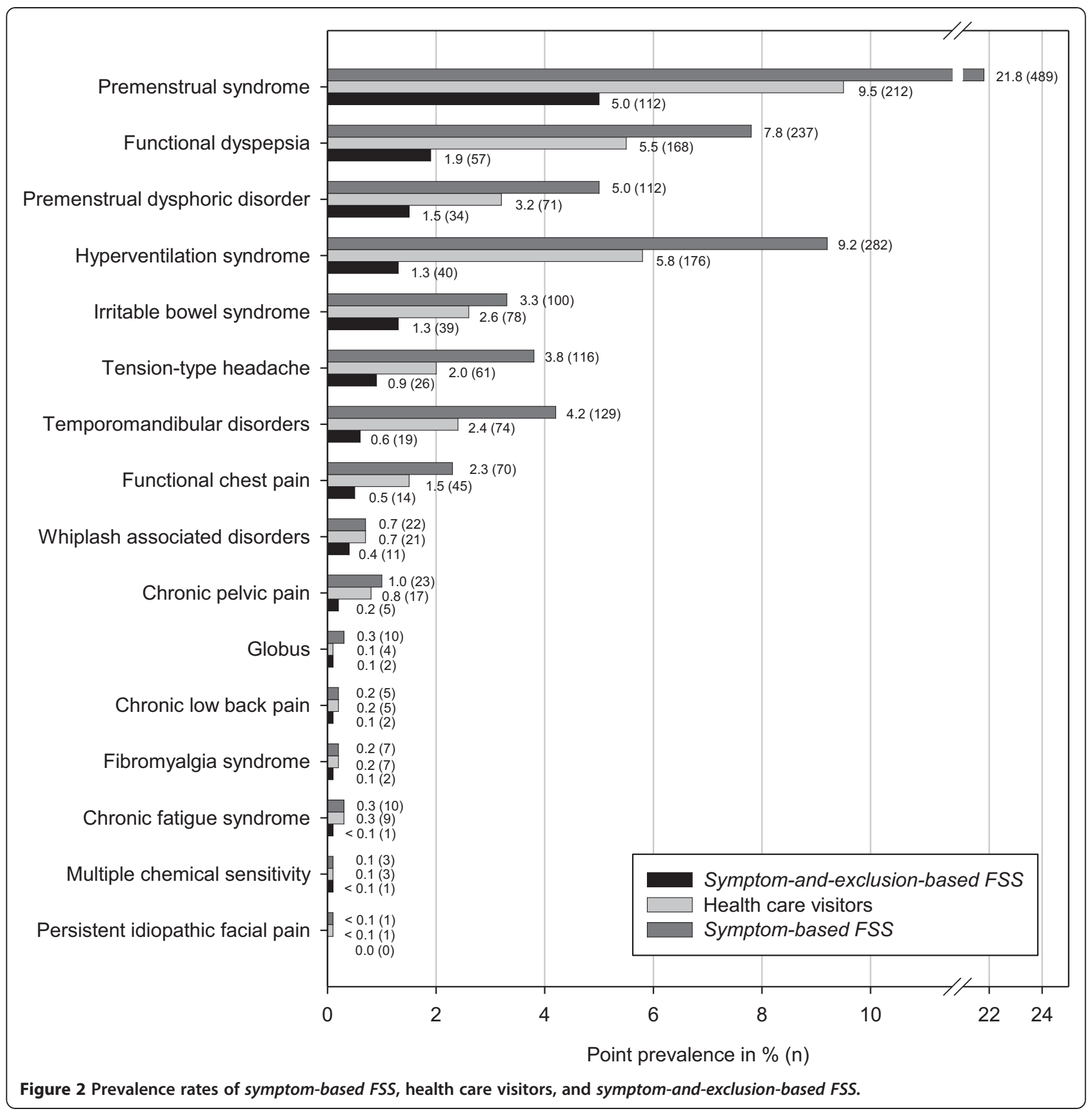

number of participants at each step of this process. For example, before vs. after medical examination by the study investigators, chronic fatigue syndrome was present in 555 vs. 43 individuals in a US-based study [24], and $7.5 \%$ vs. $1.6 \%$ in a French sample were estimated to have fibromyalgia [25]. Similarly, an in-depth look at the study by Koloski et al. [26], in which an approach comparable to our symptom-and-exclusion-based strategy was used in functional gastrointestinal disorders, reveals a more than doubled decrease in prevalence rates before vs. after the exclusion of medical illnesses. This suggests that the use of our symptom-and-exclusion-based strategy 'mimics' the diagnostic pathway of epidemiological gold standard studies adequately well. For absolute comparisons of prevalence rates obtained by this strategy with findings of other studies, the interested reader is referred to a previously published article by our group [13].

Based on our findings, we further argue that potential misattribution of somatic symptoms to a specific FSS (instead of a medical illness) artificially inflates the extent of overlap between syndromes. Only a small number of population-based studies have examined overlap between 
multiple (i.e., more than two) FSS [27-30]. On the one hand, two of these studies relied either on physician-based [27] or on symptom-based [28] diagnoses. Their finding of a substantial co-occurrence of FSS is in accordance with our finding of more than nine concomitant syndromes in our symptom-based FSS group. Interestingly, in one of these studies, the authors argue that their 'results support theories suggesting that medically unexplained conditions share a common etiology' [[27]; p. 818]. This study, as well as our finding of a considerable overlap between symptom-based diagnoses, are therefore in favor of the single syndrome hypothesis [lumpers' position; [10]]. On the other hand, in another study, a symptom-and-exclusionbased strategy was used for the diagnosis of FSS [30]. After re-analyzing their Swedish Twin Registry data, Kato et al. reported that only $2.8 \%$ of their participants were characterized by multiple FSS [30]. This percentage is in line with our finding of $4.5 \%$ of participants having at least three symptom-and-exclusion-based FSS. Based on their findings, the authors conclude that 'taken together, overlaps among the three functional somatic syndromes were not substantial' (p. 451). This study, as well as our data obtained by using the symptom-and-exclusion-based strategy, thus both lend support to the notion of the existence of multiple specific FSS instead of one single syndrome [splitters' position; [11]]. Taken together, study findings regarding the overlap between FSS seem to depend heavily on the selected diagnostic strategy, a finding which has important conceptual ramifications (one vs. many debate). Importantly, to answer the question of overlap, and whether FSS are all expressions of the same underlying phenomenon or discrete diagnoses, a different analysis strategy should be employed [see e.g., [31,32]]. This strategy would ideally combine a factor analysis with latent class analysis. Unfortunately, the hierarchical, modular structure of the herein used FFSS prevented the use of this approach in the current data set.

\section{Study strengths and limitations}

A strength of our study lies in our access to a large, nonclinical sample that was free of any healthcare-seeking bias. Nevertheless, a number of limitations need to be taken into account when interpreting our results. First, the present survey was conducted in a student sample, which cannot be considered representative of the general population. However, as outlined above, our findings are in accordance with general population-based studies, indicating potential generalizability at least to some extent. Second, our strategy of establishing diagnoses of FSS was dependent on health care visits. This led to a reduction of our sample size, and could have potentially resulted in an underestimation of 'true' prevalence rates in symptomand-exclusion-based FSS. However, accounting for medical exclusionary conditions is very likely to explain a large proportion of the decrease in prevalence rates, as mirrored by the fact that in $43.1 \%$ of cases, a medical explanation for patients' symptoms was provided by a health care professional. Third, due to the nature of a web-based data collection approach, we were unable to confirm our diagnoses through a physical examination or laboratory assessment in our participants (gold standard procedure). This might again have led to an underestimation of 'true' prevalence rates in symptom-and-exclusion-based FSS, since patients whose symptoms were caused both by an FSS and a medical condition were not counted as FSS cases in this study. In other words, we considered those individuals having a medical condition that explained their symptoms on part as non-cases. Also, some of the exclusionary medical conditions might have been incidental, with the FSS actually causing the symptoms. As illustrated above, our diagnostic strategy does, however, lead to similar decreases in prevalence rates compared to those epidemiological studies using the gold standard approach.

\section{Conclusions}

To summarize, we were able to show that including information on exclusionary medical conditions leads to a significant decrease in prevalence and overlap rates of FSS. This may call into question the validity of the findings of a number of epidemiological studies on FSS. In a next step, the validity of our symptom-and-exclusion-based strategy should be checked in FSS patients that were diagnosed by the gold standard procedure. Also, comparisons of prevalence rates as obtained by our approach with prevalence rates of self-reported and physician diagnoses would be of interest. Future studies should adopt more rigorous approaches to the study of FSS, and combine both the positive and negative criterion inherent in their definition. This is likely to enhance the clinical benefit from epidemiological findings on FSS, with the potential to guide diagnostic and, ultimately, treatment decisions.

Abbreviation

FSS: Functional somatic syndromes.

\section{Competing interests}

The authors declare that they have no competing interests.

\section{Authors' contributions}

SF contributed to the conception and design of the study, analyzed and interpreted the data and provided a first draft of the manuscript. UMN contributed to the conception and design of the study and revised earlier versions of the manuscript. Both authors read and approved the final manuscript.

\section{Acknowledgments}

SF and UMN acknowledge funding by the Swiss National Science Foundation and the Volkswagen Foundation. The funding institutions had no role in the design, collection, analysis, and interpretation of data, in the writing of the manuscript, or in the decision to submit the manuscript for publication. 
Received: 4 April 2014 Accepted: 30 September 2014

Published: 4 October 2014

\section{References}

1. Fukuda K, Straus SE, Hickie I, Sharpe MC, Dobbins JG, Komaroff A: The chronic fatigue syndrome: a comprehensive approach to its definition and study. International Chronic Fatigue Syndrome Study Group. Ann Intern Med 1994, 121:953-959.

2. Longstreth GF, Thompson WG, Chey WD, Houghton LA, Mearin F, Spiller RC: Functional bowel disorders. Gastroenterology 2006, 130:1480-1491.

3. Wolfe F, Smythe HA, Yunus MB, Bennett RM, Bombardier C, Goldenberg DL, Tugwell P, Campbell SM, Abeles M, Clark P, Fam AG, Farber SJ, Fiechtner JJ, Franklin CM, Gatter RA, Hamaty D, Lessard J, Lichtbroun AS, Masi AT, McCain GA, Reynolds WJ, Romano TJ, Russell IJ, Sheon RP: The American College of Rheumatology 1990 Criteria for the Classification of Fibromyalgia. Report of the Multicenter Criteria Committee. Arthritis Rheum 1990, 33:160-172.

4. Wolfe F, Clauw DJ, Fitzcharles MA, Goldenberg DL, Katz RS, Mease P, Russell AS, Russell IJ, Winfield JB, Yunus MB: The American College of Rheumatology preliminary diagnostic criteria for fibromyalgia and measurement of symptom severity. Arthritis Care Res (Hoboken) 2010, 62:600-610.

5. Prins JB, van der Meer JWM, Bleijenberg G: Chronic fatigue syndrome. Lancet 2006, 367:346-355.

6. Lovell RM, Ford AC: Global prevalence of and risk factors for irritable bowel syndrome: a meta-analysis. Clin Gastroenterol Hepatol 2012, 10:712-721 e714.

7. McBeth J, Jones K: Epidemiology of chronic musculoskeletal pain. Best Pract Res Clin Rheumatol 2007, 21:403-425.

8. Aaron $L A$, Buchwald D: A review of the evidence for overlap among unexplained clinical conditions. Ann Intern Med 2001, 134:868-881.

9. Rodriguez MA, Afari N, Buchwald DS: Evidence for overlap between urological and nonurological unexplained clinical conditions. J Urol 2009, 182:2123-2131.

10. Wessely S, Nimnuan C, Sharpe M: Functional somatic syndromes: one or many? Lancet 1999, 354:936-939.

11. Wessely S, White PD: There is only one functional somatic syndrome. $\mathrm{Br} \mathrm{J}$ Psychiatry 2004, 185:95-96.

12. Warren JW, Clauw DJ: Functional somatic syndromes: sensitivities and specificities of self-reports of physician diagnosis. Psychosom Med 2012, 74:891-895.

13. Fischer S, Gaab J, Ehlert U, Nater UM: Prevalence, overlap, and predictors of functional somatic syndromes in a student sample. Int J Behav Med 2013, 20:184-193.

14. Nater UM, Fischer S, Latanzio S, Ruoss D, Gaab J: FFSS - Fragebogen zur Erfassung funktioneller somatischer Syndrome [FFSS - Questionnaire on Functional Somatic Syndromes]. Verhaltenstherapie 2011, 21:263-265.

15. IHS: The International Classification of Headache Disorders: 2nd edition. Cephalalgia 2004, 24(Suppl 1):9-160.

16. Dworkin SF, LeResche L: Research diagnostic criteria for temporomandibular disorders: review, criteria, examinations and specifications, critique. J Craniomandib Disord 1992, 6:301-355.

17. Galmiche JP, Clouse RE, Balint A, Cook IJ, Kahrilas PJ, Paterson WG, Smout AJ: Functional esophageal disorders. Gastroenterology 2006, 130:1459-1465.

18. Tack J, Talley NJ, Camilleri M, Holtmann G, Hu P, Malagelada JR, Stanghellini V: Functional gastroduodenal disorders. Gastroenterology 2006 130:1466-1479.

19. Bartha L, Baumzweiger W, Buscher DS, Callender T, Dahl KA, Davidoff A, Donnay A, Edelson SB, Elson BD, Elliott E, Flayhan DP, Heuser G, Keyl PM Kaye H, Gibson P, Jason LA, Krop J, Mazlen RD, McGill RG, McTanney J, Meggs WJ, Morton W, Nass M, Oliver CL, Panjwani DD, Plumlee LA, Rapp D, Shayeviz MB, Sherman J, Singer RM, Solomon A, Vodjani A, Woods JM, Ziem G: Multiple chemical sensitivity: a 1999 consensus. Arch Environ Health 1999, 54:147-149.

20. Nickel JC, Nyberg LM, Hennenfent M: Research guidelines for chronic prostatitis: consensus report from the first National Institutes of Health International Prostatitis Collaborative Network. Urology 1999, 54:229-233.

21. WHO: The ICD-10 Classification of Mental and Behavioural Disorders - Clinical Descriptions and Diagnostic Guidelines. Geneva: World Health Organization; 1992.
22. APA: Diagnostic and Statistical Manual of Mental Disorders (4th edition, Text Revision). Washington D.C: Author; 2000.

23. van Dixhoorn J, Duivenvoorden HJ: Efficacy of Nijmegen Questionnaire in recognition of the hyperventilation syndrome. J Psychosom Res 1985, 29:199-206.

24. Reyes M, Nisenbaum R, Hoaglin DC, Unger ER, Emmons C, Randall B, Stewart JA, Abbey S, Jones JF, Gantz N, Minden S, Reeves WC: Prevalence and incidence of chronic fatigue syndrome in Wichita, Kansas. Arch Intern Med 2003, 163:1530-1536.

25. Perrot S, Vicaut E, Servant D, Ravaud P: Prevalence of fibromyalgia in France: a multi-step study research combining national screening and clinical confirmation: The DEFI study (Determination of Epidemiology of Flbromyalgia). BMC Musculoskelet Disord 2011, 12:224.

26. Koloski NA, Talley NJ, Boyce PM: Epidemiology and health care seeking in the functional GI disorders: a population-based study. Am J Gastroenterol 2002, 97:2290-2299.

27. Schur EA, Afari N, Furberg H, Olarte M, Goldberg J, Sullivan PF, Buchwald D: Feeling bad in more ways than one: comorbidity patterns of medically unexplained and psychiatric conditions. J Gen Intern Med 2007, 22:818-821.

28. Aggarwal VR, McBeth J, Zakrzewska JM, Lunt M, Macfarlane GJ: The epidemiology of chronic syndromes that are frequently unexplained: do they have common associated factors? Int J Epidemiol 2006, 35:468-476.

29. Dansie EJ, Furberg H, Afari N, Buchwald D, Edwards K, Goldberg J, Schur E, Sullivan PF: Conditions comorbid with chronic fatigue in a populationbased sample. Psychosomatics 2012, 53:44-50.

30. Kato K, Sullivan PF, Pedersen NL: Latent class analysis of functional somatic symptoms in a population-based sample of twins. J Psychosom Res 2010, 68:447-453.

31. Fink P, Schroder A: One single diagnosis, bodily distress syndrome, succeeded to capture 10 diagnostic categories of functional somatic syndromes and somatoform disorders. J Psychosom Res 2010, 68:415-426.

32. Rosmalen JG, Tak LM, de Jonge P: Empirical foundations for the diagnosis of somatization: implications for DSM-5. Psychol Med 2011, 41:1133-1142.

doi:10.1186/1471-2458-14-1034

Cite this article as: Fischer and Nater: Functional somatic syndromes: asking about exclusionary medical conditions results in decreased prevalence and overlap rates. BMC Public Health 2014 14:1034.

\section{Submit your next manuscript to BioMed Central and take full advantage of:}

- Convenient online submission

- Thorough peer review

- No space constraints or color figure charges

- Immediate publication on acceptance

- Inclusion in PubMed, CAS, Scopus and Google Scholar

- Research which is freely available for redistribution 